\title{
تأثير لغة الأم في عملية التعليم
}

\section{Dzikrul Hakim Al Ghozali \& Malia Fransisca}

Universitas KH. A. Wahab Hasbullah (UNWAHA) Tambakberas Jombang, dan Institut Agama Islam (IAI) Sunan Giri Bojonegoro

Email: $\underline{\text { adzik202@gmail.com, MALIAFRANSISCA2018@gmail.com }}$

\begin{abstract}
:
"In the process of acquisition of B1, a person has undergone a course (acquisition of language naturally), so automatically all elements of language in B1 will be attached and easily he mastered. Another case with B2, one still has to try (consciously) to master all elements of language about B2. There are several relationships between $B 1$ and $B 2$ that a person is studying, including both language equations and language differences, as well as language structure. The existence of easy in learning B2 because there are some similarities between B1 and B2. Conversely, difficulties arise in learning $B 2$ because of the differences between the two languages, which can even lead to errors. So with this please note that the influence of B1 to B2 and will always exist."
\end{abstract}

Keywords: Mother Language, Target Language, Language Learning.

اللغة ما لا تفرق بحياة الناس. وبالاصطلاح الآخر أهفا ما يشعل بالناس أنفسهم.' اللغة آلآت المواصلات،

تبليغ الفكرة والخاطرة والتعبير والتعامل بين الناس. ازدهرت مطالب العولمة وتطوير العلوم والتكنولوجية ازدهارا الذي يسبب البلد يحتاج إلى البلد الآخر. فلذلك، كانت الفطرة بين الناس ليعاملوا بينهم لكي تكفي حاجاقم ولتنمية تطور الشعوب والمصلحة العامة.

كل الشعوب عندهم التخصيص، إما بنظام الدولة وإما بالسياسية والاقتصادية والثقافية بل لغاتم. فلذلك، علينا نعرف أننا نحتاج إلى اللغات كثيرة لمعاملة الناس بعضهم ببعض. لأن شعوب البلد ليس من البلد نفسه ولكن. يكون من بلد آخر. لذلك، وجب علينا أن نعرف ونفهم اللغات الأخرى ونفهم بخلف خلفياقم المختلفة.

${ }^{1}$ A. Chaer, "Psikolinguistik: Kajian Teoritik", (Jakarta: Rineka Cipta, 2009), h. 5. 
كانت الأسباب التى تأثر بعملية تعليم اللغة كالتشجيع وتعرض الرسمي والبيئة وما إلى ذلك. ومن أحد العوام ل المجذبة على الباحث ليكشف الرسالة هي تأثير اللغة الأولى على عملية تعليم اللغة الثانية لتحقق تحصيل اكتسا ب اللغة الثانية. وكان الباحث يشرح فيما يلي.r

\section{المبحث}

\section{1 ا ت تعريف اللغة}

فأما اللغة لغة هي آلآت المواصلات. وأما اللغة اصطلاحا هي آلآت المواصلات عندها التعبيرات المعل

ومات الصحيحة. بّ يحتاج الناس إلى اللغة لبناء المواصلات بعضهم بعضا. كان الناس كالإنسان العاملة في ح

$$
\text { ياتم التى تتعلقون باللغة التى يستخدمون في تحدثهم. }
$$

هذا يناسب بالمقالة: “،إن كان الشعب كانت اللغة”. والقول الآخر: ““اينما حدث الأحداث فوجد

$$
\text { ت اللغة“’ }
$$

وصف اللغويون أن اللغة هي رمز اعطباطي الذي يستخدم بالمجتمع ليتواصلوا ويعرفوا أنفسهم. اللغة هـ ي نظام نظاميا الذي ليس يشتق بالمفرد وحدها بل بالنحو والأصوات والمعاجم أيضا.

أصل اللغة متنوعة ومتعلقة بحضارة الناس. قال Von Schlegel أن اللغات في هذا العالم لا يمكن من للغة الواحدة. أصل اللغة هي مختلفة تتعلق بالعوامل التى تسبب بتكوين تلك اللغة. وقال أيضا: ،كأينما يأتي اللغة، كان عقل الناس يسبب تطورا تاما." وبالقول الأخر، أصل اللغة من حضارة الناس في العالم.ج

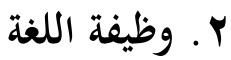

قال أهل الاجتماع اللغوي Wardhaugh عند اللغة وظيفة للمواصلات، إما باللسان وإما بالكتابة. \و اتفق Indah dan Abdurrahman به، فيقولا كما يلي:

\footnotetext{
${ }^{2}$ Ibid., h. 251.

${ }^{3}$ Indah \& Abdurrahman, “Psikolinguistik: Konsep \& Isu Umum”, (Malang: UIN Malang Press. 2008), h. 46.

${ }^{4}$ Ibid.

5 Ibid, A. Chaer, h. 30 .

${ }^{6}$ Ibid., h. 31-32.

${ }^{7}$ Ibid., h. 33.

${ }^{8}$ Ibid., Indah \& Abdurrahman..., h. 50.
} 


\section{Dzikrul Hakim \& Malia Fransisca}

أ) وظيفة اللغوية هي داخل الشخص نفسه أي استخدام اللغة ليكشف الأسئلة وعقد التقرير والتفكير والتذ كير وما إلى ذلك.

ب) وظيفة اللغوية هي العلاقة الشخصية أي أن اللغة تدل على إرادة القائل. ويعبر بكلمة الأمر والاستفها م والخبر.

قال Kinneavy أن وظيفة الأساسية اللغوية خمسة وخصوصا هي وظيفة التعبير والخبر والاكتشاف وال اقتناع والتسلية. وأما الاكتشاف هو تعبير باستخدام اللغة ليشرح الأشياء والأحوال والأمور . وأما الاقتناع هـ وسي ولهيك

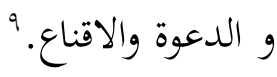

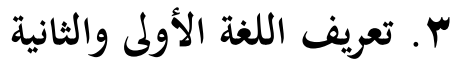

The important features that all shades of L اللغة الأولى هي اللغة التى ينال الصبي في بكوره. 1 share are that they are assumed to be languages which are acquired during early child hood, normally beginning before the age about three years بي في بداية كلامه بالبيئة شفهيا وطبيعيا.

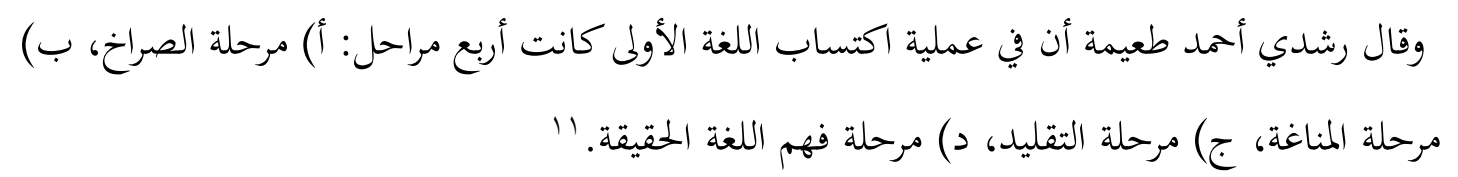
وأما اللغة الثانية هي اللغة التى تتعلم بعد اكتساب اللغة الأولى. في المعجم الكبير يشرح أن اللغة الثاني ة هي اللغة التى تفهم مع اللغة الأولى في أول عصر حياعم واجتماعا وثقافيا كلغات نفسهم. اللغة الثانية ي

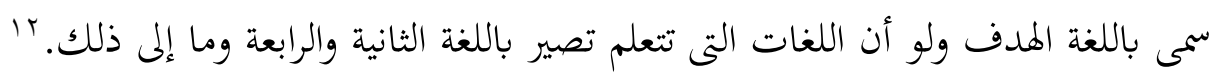

\footnotetext{
${ }^{9}$ Ibid, A. Chaer..., h. 33.

${ }^{10}$ Isnaini, Iswahyuni, Hapsari, Y. \& Dewi, “Modul Bahasa Inggris: Foreign Language Acquisition”, (Tanpa Kota: 2011, Universitas Brawijaya), h. 2.

" رشدي أحمد طعيمة، "لمرجع في تعليم اللغة العببية: للناطقين بلغات أخرى"، (مكة المكرمة: مطابع جامعة أم القرى، مجهول السنة)، ص: عی-

12 Ibid., h. 2-3. 
اكتساب اللغة الأولى يختلف بتعليم اللغة الثانية. وأما اكتساب اللغة هي عملية المستمرة في مخ الأطفا ل عند ما يكتسبون اللغة الأولى. وتعليم اللغة تتعلق بالأحداث عندما يتعلمون اللغة الثانية بعد اكتساب ال لغة الأولى. إذا....... اكتساب اللغة يتعلق باللغة الأولى وتعليم اللغة يتعلق باللغة الثانية.

\section{ع. العلاقة بين اللغة الأولى في تعليم اللغة الثانية}

كانت العلاقة بين اللغة الأولى والثانية. ومن العلاقات هي من حيث المعادلة والمفارقة في عناصر اللغو ية وهيكل اللغة. فأما المعادلة تقع بترتيب اكتساب كوضع الاستجواب والنفى والمورفيمات النحوية. وعنصو راللغوي المعين يكتسب أولا، وأما عنصور اللغوى الآخر يكتسب من بعد. بّا وأما المفارقة بين اللغة الأولى و الثانية هي أن اكتساب اللغة الأولى هو عنصور حقيقي من تطور المعريي والاجتماع للأطفال. وأما اكتساب اللغة الثانية بعد انتهاء تطور المعرفي والاجتماعى للأطفال. ومن حيث مفهوم النطق، كانت الأطفال أفهم بنطق اللغة الأولى. وأما نطق اللغة الثانية يشعر الأطفال صعبة وغير التامة. تطور Robert Lado Charles Fries الفرضية التى تسمى بالفرضية الانقباضية التى تبحث المفارقة بين اللغة الأولى والثانية. ومن المفارقة تكون سهلة وصعبة في اكتساب اللغة الثانية. توجد السهلة في تعليم ا للغة الثانية بوجود المعادلة بين اللغة الأولى والثانية. وبالعكس، توجد الصعبة في تعليم اللغة الثانية بوجود المفا رقة بين اللغة الأولى والثانية بل يلحق الأخطاء.

في الفرضية الانقباضية تقرر أن المتعلمين في اللغة الثانية ينقلون اللغة الأولى في اللغة الثانية غالبا في أف كارهم. هذا الانتقال يحدث بجميع طباقات اللغوية مثل نظام الأصوات والتركيب وترتيب الجملة. وإذا تحد ث تعليم اللغة الثانية، كان النقل الايجابي والسلبي بينهما. النقل الايجابي هو وجود المعادلة التركيبية التى تلح ق بالسهلة وأما النقل السلبي هو وجود المفارقة التركيبية التى تلحق بالصعبة في عملية تعليم اللغة الثانية.؟ عندما تعليم اللغة الثانية، كان الرجل ويخصص بالأطفال يميلين إلى اللغة الأولى لابتداء التكلم في اللغة الثانية قبل أن ينالوا اللغة الثانية. في الفرضية اللغوية الأولى التى تطور ستيفين كراسين يقول بأن: “اللغة الأو لى تستخدم لابتداء التحدث باللغة الثانية حينما لم يظهر فهم اللغة الثانية. "عندما جبر الأطفال باستخدام

${ }^{13}$ Ibid., A. Chaer..., h. 246-247.

14 Ibid, h. 247. 


\section{Dzikrul Hakim \& Malia Fransisca}

اللغة الثانية في مرحلة التعليم فيستخدم الأطفال المفردات في اللغة الأولى وتركيبها. اعط الفرصة للأطفال لينا لوا الأشياء ذا المعنى وهو ينقص استيعاب الموقف. ولذلك، فهم اللغة الثانية يتطور في نفس الوقت. رأى Ellis على المتعلمين يؤمنون أن اللغة الأولى تؤثر على عملية لفهم اللغة الثانية. اتفق Dulay مع ذلك ويقول بأن اللغة الأولى تصير مزعج في عملية تعليم اللغة الثانية.

وتلك واقعة لأن متعلم اللغة الثانية عمدا أو غير عمد ينقل عناصر اللغة الأولى باللغة الثانية عندما ي

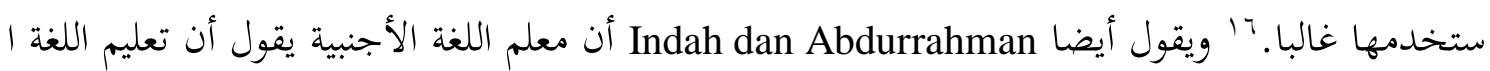
لبعيدة تحتاج إلى طوال الوقت من تعليم اللغة القريبة من حيث المفارقة باللغة الأولى، مثل: متكلم اللغة الإن جليزية يحتاج إلى طوال الوقت لتعليم اللغة الصينية من تعليم اللغة الإسبانية. لا ومن الأقوال فظهرت الأسئلة في قلوبنا، ماذا نعمل؟ هل نستطيع أن نحتل الوسوسة في اللغة الأولى بتعليم اللغة الثانية؟ وهل نستطيع أن ن نقصها؟ كانت نظريتان لإجابة من تلك الأسئة:

أ) نظرية الاستجابة التحفيزية التى يقول مجموعة السلوكية اللغة هي حاصل السلوك من الاستجابة التحفيزية فصار عندما يريد المتعلم أن يكثر باستخدام النطق، فكثر بتلقاء التحفيز . فلذلك، كانت البيئة تؤثر قوية في عملية تعليم اللغة الثانية. وهم يقولون أيضا بأن اكتساب اللغة هو عملية العادة أي إن كثر تحفيز الأ شياء التى تؤتى إلى المتعلم فكثر اكتساب اللغة الثانية. وبالعكس، عندما المتعلم لم يلق التحفيز من الخار ج فلم يستطع المتعلم أن يعمل الأنشطة المردودة.^` تأثير اللغة الأولى في شكل النقل عندما يتكلم في ال لغة الثانية أكبر التأثير عندما لم يستمر المتعلم باللغة الثانية استمرارا. وهذا التأثير استيعاب في نفس المتعل م ولكن بوجود العادة المستمرة بإعطاء التحفيز المستمر ينقص تأثير اللغة الأولى على عملية تعليم اللغة الئرسي لثانية. ب) نظرية المختلفة هي يعين حاصل تعليم اللغة الثانية بأحوال اللغة المقدمة التى تتعلم قبله.9 سوى ذلك، أحد العوامل التى تؤثر تعليم اللغة الثانية هو عامل اللغوية هي يتعلق باختلاف اللغة الأولى والثانية من نا سابل

\footnotetext{
${ }^{15}$ Ibid, h. 249.

${ }^{16}$ Ibid, A. Chaer..., h. 256.

${ }^{17}$ Ibid, Indah \&Abdurrahman..., h. 84.

${ }^{18}$ Ibid, A. Chaer..., h, 256-257.

${ }^{19}$ Ibid, h. 257.
} 
حية النطق والتركيب ونطط الخطاب. ‘ُ استنبط Bananthy أن أكبر حال اللغة المتعلمة أي اللغة الأولى فأكبر صعبة لفهم اللغة الثانية قال Bananthy أن في تعليم اللغة الثانية، بمعرفة اللغة الأولى تساعد على

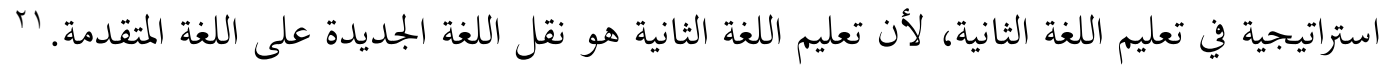

\section{هـ النقل والثدخل اللغوي في اللغة الأولى والثانية}

في الباب المتقدم ييحث في العوامل في اللغة الأولى التى تؤثر في تعليم اللغة الثانية، أن اللغة الأولى تزعج استخدام اللغة الثانية. وكان المتعلم يميل بنقل عناصر اللغة الأولى في اللغة الثانية. يسمى Chaer في دراسته بالتدخل والاختلاط والأخطاء. فطبعا، باستمرار كفاءة اللغة الثانية، ينقص الاستخدام والعملية في عناصر ا للغة الأولى بمرور الوقت. التدخل اللغوي هو ادخال عناصر اللغة في اللغة الأخرى تسبب انتهاك بقواعد ال لغوية الثانية من ناحية الأصوات والتراكيب والمعاجم والدلالة. في عملية التدخل اللغوية تحدث النقل هو اس تخدام قواعد اللغة في اللغة الأخرى ولكن يقول نباة من ناحية النظرية ما يكون كفاءة اللغة الثانية خير من

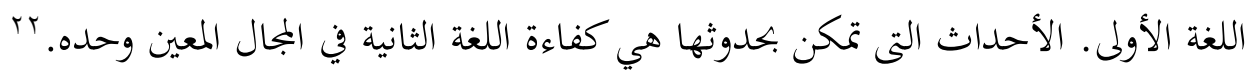

في عملية اكتساب اللغة الثانية تحدث الاستيعاب. اكتساب اللغة الأولى يستمر في الطفل حتى أواخر العصور لاكتساب اللغة الأولى قليلا فقليلا فوجد الاستيعاب في عملية اللغة الأولى. الاستيعاب يقرب بالا ستيلاء وهو يستمر في كفاءة اللغوية مثل الدلالة والأصوات والصرف والمعاجم بغير عمد. يحدث التدخل اللغوي بين اللغة الأولى والثانية يحتوى على كل شيء الذي يتعلق باللغة. فأما يلي هو الأمثلة في التدخل اللغوي:

$$
\text { أ) التدخل اللغوي في الأصوات الفونولوجيا }
$$

عندما يقول المتعلم المفردات ينطق من اللغة بتدخل الأصوات من اللغات الأخرى. المثل: عندما ناطق اللغة

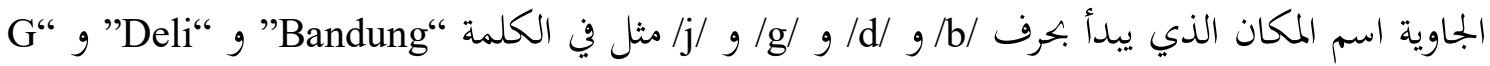

20 A. S. Ghazali, "Pembelajaran Keterampilan Berbahasa: dengan pendekatan komonikatifinteraktif", (Bandung: PT Refika Aditama, 2010), h. 126.

${ }^{21}$ Ibid, A. Chaer..., h, 257.

22 Ibid, h. 261.

${ }^{23} \mathrm{Ibid}$, h. 261-263. 


\section{Dzikrul Hakim \& Malia Fransisca}

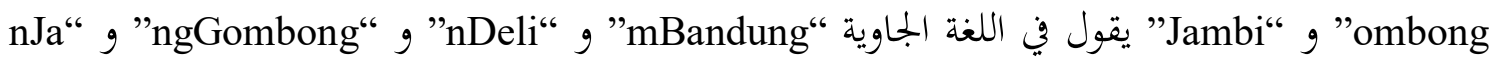
."mbi

$$
\text { ب) التدخل اللغوي في المورفيمات }
$$

يحدث التدخل اللغوي في المورفيمات عندما يدخل الزوائد في الكالام. وذلك يحدث بسبب الانحراف التركي

$$
\text { ب ب بن اللغة الثانية والأولى. }
$$

" terpukul " / "kepukul " المثل:

" dipindahkan " / " dipindah"

" peneonan " / " neonisasi "

" bertanya " / "menanyai "

المثل الآخر في اللغة الهولندي والإنجليزية وجدنا اللاحقة، فكثر ناطق اللغة الإندونيسيا الذين يستخدمون في تركيب الكلمات في اللغة الإندونيسيا مثل: tendanisasi, turinisasi وذلك الشكل يسمى بانحراف من ح يث المورفيمات في اللغة الإندونيسيا. لأن فيها موجودة الزوائد "pe-an" لشكل الاسم. إذا، الشكل الص حيح هو penendaan, penurian المثل الآخر عن استخدام شكل الكلمة ketabrak, kejebak, dan ke. kecilan في اللغة الإندونيسيا هم من التدخل اللغوية. وذلك الشكل من اللغة الجاوية وهجة جاكرتا فأما ا لشكل الصحيح هم tertabrak, terjebak, terlalu kecil.

$$
\text { ج) التدخل اللغوي في بناء الجملة }
$$

التدخل اللغوي بتركيب اللغة الأولى في اللغة الثانية مثل: طلاب الإندونيسيون الذين يتعلمون اللغة العربية ي قولون الكتاب هذا جديد. ومثل الآخر من ثنائي اللغة “الجاوية-الإندونيسيا” و “سوندا- الإندونيسيا” م ثل صوت الجملة:

- Disini toko laris yang mahal sendiri

${ }^{24}$ Nababan, “Sosiolinguistik Suatu Pengantar", (Jakarta: PT Gramedia Utama, 1984), h. 123-124.

47 An-Nas: Jurnal Humaniora, Vol. 3, No.1, 2019 
تلك الجملة تستخدم بتركيب اللغة الجاوية وأصلها: "ing kene toko laris sing larang deweN".

- Surat itu telah dibaca oleh saya

تلك الجملة من تدخل اللغوي الإندونيسيا باللغة سوندا أو أصلها: "eta surat geus dibaca ku kuring

$$
\text { د) التدخل اللغوي الثقاين }
$$

ظهر هذا التدخل باللغة التى تستخدم ناطق ثنائي اللغة. ومن ذلك المنطوق، ظهر عناصر الأجنبية التى يعق ب بناطق ليعبر الأحداث أو الخبرة الجديدة.

في الوقت الحاضر، كثير من الإندونيسيين يستخدمون المعاجم الأجنبي مثل اللغة الإنجليزية والعببية وما إلى ذ لك. هو الذي نقل العمد بعلتين: أ) لجهل المعادلة في اللغة الإندونيسيا، ب) لهيبة بأنه عالم. ويقول نباة أي ضا أن استخدام المعاجم الأجنبي ليس من النقل لأن اللغة الأجنبي هي ليس من اللغة الأولى للمتعلم. بr

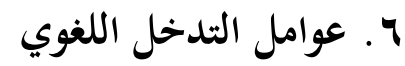

سوى اتصال اللغوي، يقول Weinrich أن العوامل التى تسبب بالتدخل اللغوي وهي: أ) ثنائي اللغة للناطق

هو أسس الذي يسبب التدخل اللغوي والعوامل الأخرى من اللغة الأصلية، إما بلغة المناطق وإما بلغة الأج نبية. وهو الذي يحدث لأن يتصل اللغة بين ناطق ثنائي اللغة والحاصل ظهر التدخل اللغوي. ب) ناقص الولاء من ناطق ثنائي اللغة على ناطق اللغة الأولى يميل بسلوك السلبية. هذا الذي يسبب بأباء قواعداللغة الثانية التى تستخدم وأخذ العناصر من اللغة الأولى التى يكافئ الناطق غير تركيب. وهذا كيعقب بظهور التدخل اللغوي في اللغة الثانية التى تستخدم الناطق إما منطوقا وإما كتابيا. ج) لمم يكف المفردات للناطق اللغة الثانية

27 Suwito, "Pengantar Awal Sosiolinguistik Teori dan Problema", (Surakarta: Henary Cipta, 1985), hal 150. 


\section{Dzikrul Hakim \& Malia Fransisca}

كانت ثروة اللغوية تحدد بتعبير الحياة في المجتمع مع شكل الحياة المعروفة. فلذلك، عندما يعامل المجتمع بأسا

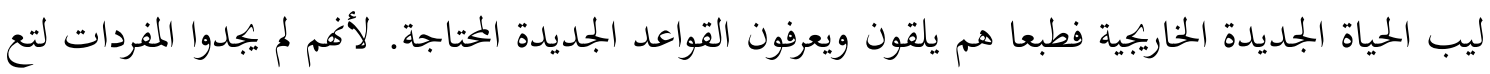

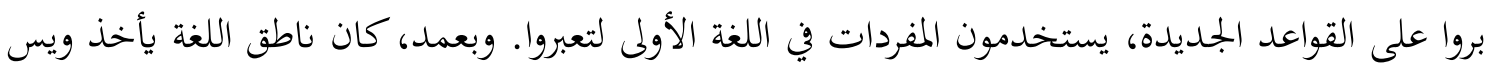
تعير المفردات في اللغة الأولى لتعبر القواعد الجديدة. ولهذه العوامل، يميل بحدوث التدخل اللغوي. التدخل اللغوي الذي يظهر لاحتياج المفردات الجديدة، يميل الناطق بعمد الكلام في حدوثه. كانت المفردا ت الجديدة التى بتد في التدخل اللغوي أسرع الاختلاط لأن العناصر محتاجة لكثرة الثروة اللغوية على الناط

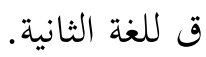

$$
\text { د) إخفاء المفردات التى نادرا باستخدامها }
$$

كانت المفردات اللغوية التى نادرا باستخدامها إخفاء بمرور الزمان. وعندما هي تحدث. فالمفردات التى المتع لقة بها يقلل بنفسها. وعندما تلك اللغة توجه بالقواعد الجديدة الخاريجية، من الناحية تنفعها بأخذ المفردات التى تخفى و من الناحية الأخرى تسبب بالتدخل اللغوي وهو الأخذ أو الاستعار المفردات الجديدة من الل

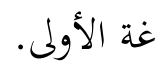

$$
\text { هـ) احتياج المرادف }
$$

المرادف في استخدام اللغوي له الوظيفة المهمة فهي كمتنوعة في اختيار الكلمة لاجتناب استخدام الكلمة ا لمعادلة المستمرة التى تعقب بالملل. وبوجود مرادف الكلمة، كان الناطق عنده تدخل اللغوي في شكل الأخذ أو الاستيعار في اللغة الثانية. فلذلك، احتياج المفردات التى عندها المرادف تحث لظهور التدخل اللغوي.

$$
\text { و) وجود العادة في اللغة الأولى }
$$

كان وجود العادة في اللغة الأولى على اللغة الثانية التى تستخدمها، تحدث لنقص المراقبة اللغوية ولنقص ال كفاءة على اللغة الثانية. وهو يحدث في ثنائي اللغة الذي يتعلم اللغة الثانية، إما اللغة الوظيفة وإما اللغة الأ جنبية. في استخدام اللغة الثانية، كانت اللغة الأولى تنقص أحيانا. لأن ثنائي اللغة الذي عندما التكلم أو الفئل لكتابة باستخدام اللغة الثانية فظهرت اللغة الأولى التى أقدم في تعريفها وفهمها.بی

${ }^{28}$ Lihat:http://pusatbahasaalazhar.wordpress.com/hakikat-hakiki-kemerdekaan/interferensi-dan-integrasi/ (Selasa: 26 Jan 2018) 


\section{الاستنباط}

اللغة هي آلآت شفهية التى تستخدم باتصال إما بتعبير الفكرة والتفكير والتعبير وإما بنقل المعلومات إلى الشخص. وغالبا، ماكانت اللغة مستقلة عن حياة الناس. عندما يكون الاتصال فتستخدم وتتطور اللغة.

اللغة الأم أو تسمى باللغة الأولى هي اللغة اللتى تكتسب أول مرة على الأطفال في طفلهم. كانت هذه العملية ت سمى بـ acquisition أي اكتساب اللغة بغير عمد وبطبيعية. عندما يعبر الطفلة إرادته بشكل اللغوي إلى أمه أو بي كتئه. هناك اللغة الأولى تكتسب.

واللغة الثانية هي اللغة التى تتعلم عندما يملك الشخص اللغة الأولى كانت عملية اللغة الثانية هي التعليم. وهذ ا........ لأن الشخص لم تتعلمها طبيعيا إلا بوجود تأثير التعليم تعليما. في تعليم اللغة الثانية لم تستطع الشخص أ ن يترك اللغة الأولى لأن تعليم اللغة الثانية تستوى بقبول اللغة الجديدة على اللغة التى تكتسب قبله. فهذه............... يمكن بتأثير عناصر اللغة الأولى إما من ناحية الأصوات وبناء الكلمة والمورفيمات وإما بالمعاجم على عناصر اللغة إل لثانية وهذا التأثير يكون نقلا وتدخلا اللغوي بين اللغتين.

وكانت العلاقات بين اللغة الأولى والثانية التى تتعلم الشخص إما بالمعادلة والمفارقة عن عناصر اللغوية، وإما بتركيبه اكانت سهلة في تعليم اللغة الثانية لأن تكون المعادلة بين اللغة الأولى والثانية. وبالعكس، ظهرت المفارقة في تعليم هما لتكون المفارقة بينهما التى تظهر الأخطاء.

النقل والتدخل اللغوي هما عملية الناطق للغة الأولى لاستخدام اللغة الأولى ولتعليم اللغة الثانية. إذا، عناصر اللغو ية التى تحتوى من المعادلة والمفارقة عندما تأثير على تحصيل تعليم اللغة الثانية. إن كانت المعادلة أكثر فأسهل في تع ليم اللغة الثانية، وبالعكس إن كان المفارقة أكثر فأصعب في تعليمها.

\section{المراجع}

\section{المرجعان العربيتان}

دمياطي، عفيف الدين. "عحاضرة في العلم الاجتمعاعية". سوراباياي. مطبعة دار العلوم اللغوية. • 1 • ؟. 


\section{Dzikrul Hakim \& Malia Fransisca}

طعيمة، رشدي أحمد. "المرجع في تعليم اللغة العربية: للناطقين بلغات أخرى". مكة المكرمة. مطابع جامعة

$$
\text { أم القرى. مجهول السنة. }
$$

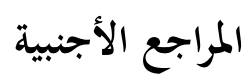

A. Chaer. "Psikolinguistik: Kajian Teoritik". Jakarta: Rineka Cipta. 2009.

A. S. Ghazali. "Pembelajaran Keterampilan Berbahasa: dengan pendekatan komonikatifinteraktif'. Bandung: PT Refika Aditama. 2010.

http://pusatbahasaalazhar.wordpress.com/hakikat-hakiki-kemerdekaan/interferensi-danintegrasi/

Indah \& Abdurrahman. "Psikolinguistik: Konsep \& Isu Umum”. Malang: UIN Malang Press. 2008 .

Isnaini, dkk. "Modul Bahasa Inggris: Foreign Language Acquisition". Tanpa Kota: Universitas Brawijaya. 2011.

Nababan. “Sosiolinguistik Suatu Pengantar". Jakarta: PT Gramedia Utama. 1984.

Suwito, "Pengantar Awal Sosiolinguistik Teori dan Problema”. Surakarta: Henary Cipta. 1985. 